\title{
Creating an end-to-end information flow to enhance last-mile logistics
}

\author{
Otto Petrovic $^{1}$, Michael Harnisch ${ }^{2}$, Thomas Puchleitner ${ }^{2}$ \\ ${ }^{1}$ University of Graz, Institute of Information Science and Information Systems \\ ${ }^{2}$ University of Graz / evolaris next level GmbH
}

\begin{abstract}
The advancements in communication technology are providing the opportunity to integrate an end-to-end information flow into the traditional last-mile logistics process. The utilization of collected and analyzed information can create lock-ineffects for senders and recipients and thus enhance last-mile logistics.
\end{abstract}

Keywords: last-mile logistics; information flow; mobile communication

\section{Introduction}

Logistics providers are continuously challenged to improve their efficiency in deliveries. Especially on the last-mile, advancements inherit a huge cost-saving potential, because between $13 \%$ and $75 \%$ of all expenditures related to logistics arise on the last-mile [1]. With the broadening of e-Commerce activities, also the number of shipments increases constantly by around $4 \%$ per year [2], which is another task that logistics providers need to manage successfully in order to stay competitive.

Beside logistics, the area of mobile communication experienced significant developments from technological and societal perspectives [3]. Smartphones enable individuals to interact without limitation of time and location.
The integration of the advances in communication into the processes of logistics provider on the last-mile, offers various opportunities for applications on the last-mile. If a location- and timeindependent information flow between all parties of the logistics process could be established successfully (end-to-end information flow), it is conceivable that enhancements in terms of efficiency and cost-improvements occur.

\section{Create the end-to-end information flow for last-mile logistics}

In communication technology, the transformation from location-oriented services to person-oriented services was already seen, by the progress from fixedline telephone calls to mobile telephone calls. Individuals are not calling "a place" anymore but "a person". This shift will also be a main opportunity for logistics providers in the next years. The establishment of an end-to-end information flow to enable a constant time- and location-independent interaction with the customer provides many opportunities in regard to the basic physical service. Examples would be the reaction on a scheduled shipment delivery in form of an individual re-routing or delay of the planned delivery.

\subsection{Bits and atoms in logistics}

Logistics was long a business, which relied heavily and solely on atoms. Since 
the integration of information between the sender and the logistics provider started to emerge, the business is more and more focusing on bits. In this context bits on the one side represent information, which is gathered, exchanged and analyzed by the partners of the logistics process. Atoms on the other side describe physical services, or the 'traditional way of conducting business', for example the explicit delivery of a shipment to the customer. [4]

The main advantage of bits over atoms is that their number is not fixed in a business. Companies are able to create and utilize bits, introduce new information and connect this data basis with applications in the field to stimulate efficiency and enhance last-mile logistics.

\subsection{Utilization of information}

The integration of information between all participants of the logistics process is now at a stage, where the focus falls on the customer and the shipment itself. This is what we call, the third phase of utilization of information in logistics, which is represented by an end-to-end integration of an interactive information flow (see Figure 1).

By utilizing smartphones and mobile applications, customers and all other in- volved parties are enabled to participate in the information exchange. The created information flow raises the efficiency of the delivery process on the last-mile.

\subsection{Information and business models}

The generated and analyzed information can be utilized to optimize and personalize the delivery process with respect to the customer. Naturally, such personalized services, which are harmonized with the individuals' preferences and needs, are advantageous for the customer and thus enhance the physical service.

Therefore lock-in-effects can arise which include financial switching costs [6] or search costs [7]. These effects result in a reinforcing feedback-loop [8], which is expressed by more usage interest if more individuals are using the information flow.

The arising end-to-end information flow creates a situation, where the logistics provider is not only able to generate personalized services for the recipients, but due to the large information base - also for the senders. In the medium term, the created information flow can thus also lock-in the senders, because the competitors are not able to generate a similar information base, once the customer as recipients are locked-in.

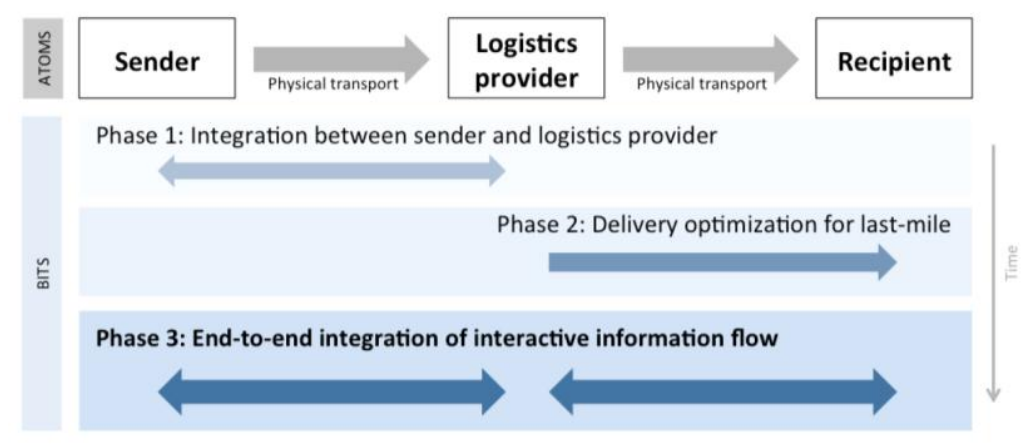

Fig. 1 Utilization of information in logistics [5] 


\section{Processing information in last-mile logistics}

Logistics provider, sender and recipient are the three involved parties within lastmile logistics. While each of these three has different needs for information and its exchange, all three share the same interest in increased efficiency regarding the exchange of shipping-related information. Recipients demand fast delivery and information regarding the current location of the shipment, whereas senders require interfaces to logistics providers for easy and flawless data integration. Logistics providers as carrier of the physical good are positioned between these two parties and therefore have an opportunity to create lock-in-effects in terms of shippingrelated communication. One key trigger is the fulfillment of senders and recipients needs regarding information processing. With an increase of senders and recipients to use a service, it continuously gains benefits which leads to lock-in effects. By providing specific benefits for both parties regarding their needs a gain on applicable information emerges.

\subsection{Integrating senders}

Regardless if the intention of the sender is business orientated or for private purposes, an effort to prepare and send a shipping is made. Services to minimize this effort fulfill a need for efficiency and are therefore beneficial for senders. Logistics providers like UPS or Australian Post allow their customers to directly integrate their shipment services into commercial platforms as eBay. 'Click and Send' [9] by Australian Post supports the linking of eBay and Australian Post accounts to organize and label all shipments and finally dispatch them by Australian Post as logistics provider. By the integration of the recipient via eBay or additionally offered services by the logistics provider an endto-end information flow is established.

\subsection{Integrating recipients}

Private e-commerce customers who occasionally receive shipments desire information regarding the delivery. Typical solutions are track and trace services that provide detailed updates on the shipments current location and expected delivery times. The application 'Slice' [10] extends the functionality of classic track and trace apps by an automatism to integrate any shipping notification, sent via email of various e-commerce providers (see Figure 2).

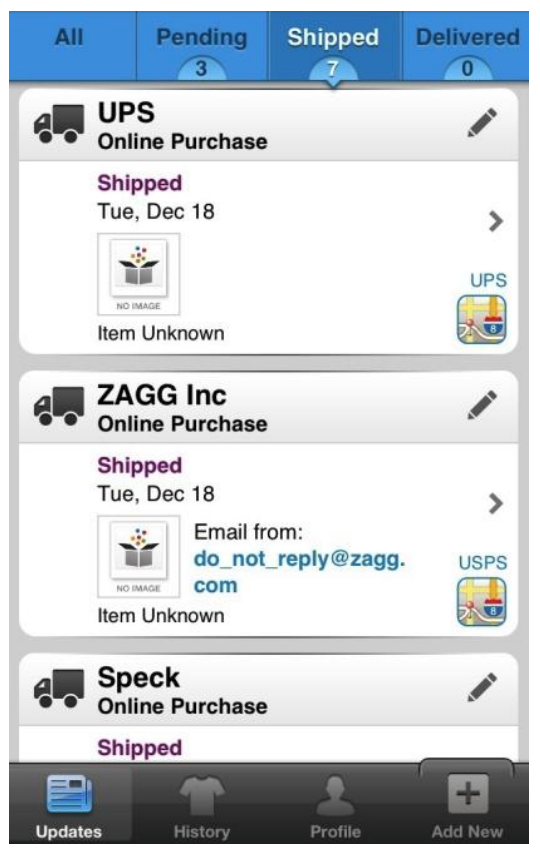

Fig. 2 Application "Slice" aggregates existing data [11]

Within their platform many beneficial features for end customers simplifies order tracking and shipping handling. Slice provides a helping feature set by allowing users to automatically process shoppingrelated emails to create a new set of information out of existing data. In this case no new information is created but already existing ones are represented in a 
user-friendly way. The service provider thereby gathers new information, which can be utilized to offer new services to sender and recipient. Again, logistics providers are in a convenient position for such a realization due to their database that already contains data related to the transportation. By connecting both existing datasets, new tracking information without any necessity for manual linking by the user can be provided.

\subsection{Interaction between senders and recipients}

E-commerce platforms usually inform buyers on actions regarding their purchase. Depending on the platform this information usually ends with the handover to the logistics provider as the provider performs all following actions. To overcome this interruption of information exchange last-mile logistics providers start their own platform where information from sender is integrated and recipients find one single contact point for all delivery relevant interactions. The mobile App "FedEx Mobile" [12] allows senders to directly send information to saved contacts. FedEx therefore fosters access by senders and recipients, which facilitates end-to-end interaction.

\section{Conclusion}

By providing beneficial services for senders as well as recipients new potentials for flawless end-to-end information flow arise. Last-mile logistics providers operate right between these two parties which allow them to use their established physical connection function for the application of new information based services. To generate lock-in-effects in form of a reinforcing feedback-loops the participation of senders and recipients is essential. The presented use cases showed ways to integrate both parties to enable an end-to-end flow of information, which therefore supports lock-in-effects.

\section{References}

[1] R. Gevaers, E. Van de Voorde, T. Vanelslander, "Characteristics and Typology of Last-Mile Logistics from an Innovation Perspective in an Urban Context", Universiteit Antwerpen, 2010.

[2] K. Esser, J. Kurze, "Wirtschaftliche Bedeutung der KEP-Branche: KEPStudie 2012 - Marktanalyse”, Bundesverband Internationaler Express- und Kurierdienste e.V. (BIEK), 2012.

[3] O. Petrovic, M. Harnisch, T. Puchleitner, „Mobile Kommunikation als ubiquitäres Phänomen“, U. Bechmann, C. Friedl (eds.), Mobilitäten, pp. 73-91, 2012.

[4] N. Negroponte, „Being digital“, Knopf, 1995.

[5] O. Petrovic, M. Harnisch, T. Puchleitner, "Opportunities of mobile communication systems for applications in last-mile logistics", in Review, 2013.

[6] C. Shapiro, H. Varian, "Information Rules, Harvard Business School, 1999.

[7] G. Zauberman, "The Intertemporal Dynamics of Customer Lock-In", Journal of Consumer Research, vol. 30, no. 3, pp. 405-419, 2003.

[8] P. Senge, "The fifth discipline: the art and practice of the learning organization”, Broadway Business, 1990.

[9] http://auspost.com.au/parcelsmail/linking-your-ebay-account.html

[10] http://www.slice.com

[11] http://cdn.gottabemobile.com/wpcontent/uploads/2012/12/Slice-LastMinute-holiday-shopping-sanity.jpg

[12] https://play.google.com/store/apps/ details?id=com.fedex.ida.android\& $\mathrm{hl}=\mathrm{en}$ 